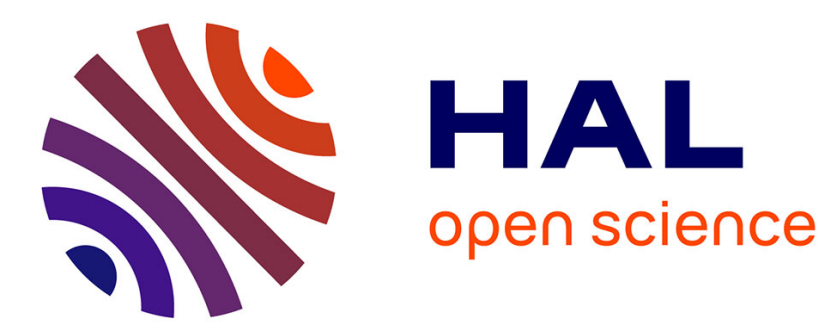

\title{
Stability and control of transversal oscillations of a tethered satellite system
}

\author{
Fabrizio Vestroni, Angelo Luongo, Monica Pasca
}

\section{To cite this version:}

Fabrizio Vestroni, Angelo Luongo, Monica Pasca. Stability and control of transversal oscillations of a tethered satellite system. Applied Mathematics and Computation, 1995, 70 (2-3), pp.343-360. hal-00801990

\section{HAL Id: hal-00801990 \\ https://hal.science/hal-00801990}

Submitted on 18 Mar 2013

HAL is a multi-disciplinary open access archive for the deposit and dissemination of scientific research documents, whether they are published or not. The documents may come from teaching and research institutions in France or abroad, or from public or private research centers.
L'archive ouverte pluridisciplinaire HAL, est destinée au dépôt et à la diffusion de documents scientifiques de niveau recherche, publiés ou non, émanant des établissements d'enseignement et de recherche français ou étrangers, des laboratoires publics ou privés. 


\section{Stability and Control of Transversal Oscillations of a Tethered Satellite System}

Fabrizio Vestroni and Angelo Luongo

DISAT-University of L'Aquila

L'Aquila, Italy

and

Monica Pasca

Department of Structural and Geotechnical Engineering

University of Rome "La Sapienza"

Rome, Italy

Transmitted by F. E. Udwadia

\section{ABSTRACT}

The tethered satellite system is characterized by weak nonlinearities but it practically works in conditions of internal resonance which produces unstable oscillations. The effect of a longitudinal control force is investigated. Since the displacement component in the orbit plane is always present in the motion due to the nonlinear coupling, the control force is assumed depending only on this component and also when a prevailing out-of-plane oscillation is considered. The harmonic balance method and numerical solutions of amplitude modulated equations are used to obtain stationary and nonstationary oscillations, respectively; the Floquet theory is followed in the stability analysis. The assumed control force is shown to be effective in reducing the primary and secondary instability regions of oscillations perturbed by internally resonant disturbance components.

\section{INTRODUCTION}

Research on the behavior of the Tethered Satellite System (TSS) has received considerable impulse from the scheduled missions that envisage its use. The papers that have appeared in dedicated meetings and journals deal with various research objectives, mainly in the field of linear dynamics 
and using simple models [1-5]. A number of studies have been devoted to the control of the system's stability and dynamics in the station-keeping configuration and during deployment and retrieval of the satellite from the orbiter [6-8].

Previous studies have revealed weak nonlinearities in the string-satellite system $[9,10]$. However, the presence of quadratic nonlinearities and the sequence of the flexible mode frequencies are such that multiple simultaneous internal resonance conditions occur. For these peculiar characteristics, the TSS exhibits some nonlinear aspects similar to those of strings, cables, and cylindrical shells [11-14]. Due to the internal resonance phenomena, transversal oscillations are found to be unstable, precisely because modes that are initially absent in the base oscillation can grow, irrespective of the smallness of perturbation, with a typical exchange of energy among the modes excited in the motion $[15,16]$.

The present paper studies the effects of longitudinal control force on the stability of motion in internal resonance condition. The control force is assumed to depend on transversal displacements of the string end. For the planar transversal oscillations perturbed out-of-plane of the orbit, the evolution of the motion for stable and unstable solutions is studied and compared with the results obtained for the uncontrolled case.

\section{EQUATIONS OF MOTION}

A suitable model to study the tethered satellite system is based on the following hypotheses: the shuttle describes an equatorial undisturbed circular orbit with constant angular velocity $n$ and radius $a$; the tether is an elastic continuum with mass density $\mu$ and elastic stiffness $E A$; the orbiter and the satellite are modeled with two point masses $(M)$ and $(m)$, respectively. In particular, the mass of the shuttle is much larger than those of the satellite and the tether and, consequently, the center of mass of the system can be consider to coincide with the orbiter center of mass.

Let consider two coordinate systems: the first $(O X Y Z)$ is a fixed reference frame with its origin in the centroid of the earth, the second (oxyz) is a rotating reference frame with the origin at the shuttle center of mass as in Figure 1. The $x$ axis is aligned with the local vertical, the $y$ axis with the tangent to the trajectory, and the $z$ axis with the orbital velocity vector. The motion can be described by the displacements $u(s, t), v(s, t)$, $w(s, t)$ in the $x, y$, and $z$ directions, respectively, measured with respect to the static straight configuration $x_{0}(s)$, where $s$ is a curvilinear abscissa along the tether. 


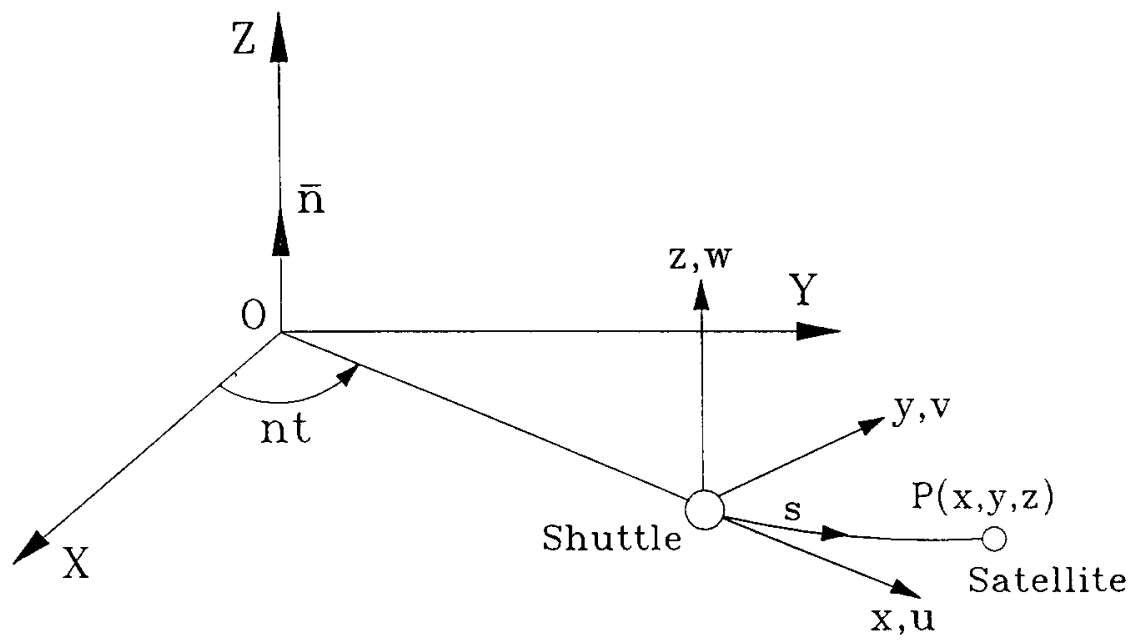

FIG. 1. Tethered satellite system and reference frames.

According to these assumptions, the equations of motion have been derived in [9] by means of a Lagrangian approach. They are here modified to introduce a longitudinal control force which is assumed proportional to the in-plane transversal velocity $\dot{v}_{e}$ of the satellite tether end $(s=\ell)$. This force $F_{c}(t)=-K_{c} \dot{v}(\ell, t)$ appears in the longitudinal equation.

The nonlinear governing equations are expanded in Taylor series up to the third order. It has been observed [15] that in correspondence with prevailing transversal oscillations, which occur in the lower range of the natural spectrum of the system, some terms in the longitudinal equations can be neglected and an integrodifferential relation among $u, v$, and $w$ is obtained. This relation makes it possible to describe the motion by only two integrodifferential equations in the transversal displacements $v(s, t)$ and $w(s, t)$. The same occurs when a linear control force depending on tranversal displacements is introduced. The equations of motion then read

$$
\begin{aligned}
& \ddot{v}-\left[f(s) v^{\prime}\right]^{\prime}-3 \xi x_{0} v-\int_{0}^{s}\left(v^{\prime 2}+w^{2}\right)^{\bullet} d s-\left[\frac{2}{\gamma} \dot{v}_{e} v^{\prime}-v^{\prime} \int_{1}^{s} 2 \dot{v} d s\right]^{\prime} \\
& +k_{c} \dot{v}_{e} v^{\prime \prime}=0 \\
& \ddot{w}-\left[f(s) w^{\prime}\right]^{\prime}+\left(1-3 \xi x_{0}\right) w-\left[\frac{2}{\gamma} \dot{v}_{e} w^{\prime}-w^{\prime} \int_{1}^{s} 2 \dot{v} d s\right]^{\prime}+k_{c} \dot{v}_{e} w^{\prime \prime}=0
\end{aligned}
$$


with relevant boundary conditions

$$
\begin{aligned}
& v(0, t)=w(0, t)=0 \\
& \ddot{v}_{e}+\gamma f(1) v_{e}^{\prime}-3 \xi x_{0}(1) v_{e}-\int_{0}^{1}\left(v^{\prime 2}+w^{2}\right)^{\bullet} d s \\
& \quad+2 \dot{v}_{e} v_{e}^{\prime}-\gamma k_{c} \dot{v}_{e} v_{e}^{\prime}=0 \\
& \ddot{w}_{e}+\gamma f(1) w_{e}^{\prime}+w_{e}\left(1-3 \xi x_{0}(1)\right)+2 \dot{v}_{e} w_{e}^{\prime}-\gamma k_{c} \dot{v}_{e} w_{e}^{\prime}=0,
\end{aligned}
$$

having introduced the dimensionless parameters

$$
\alpha^{2}=\frac{\mu n^{2} \ell^{2}}{E A} \quad \gamma=\frac{\mu \ell}{m} \quad \xi=\frac{\ell}{a} \quad k_{c}=\frac{K_{c}}{\mu n \ell}
$$

where $\ell$ is the tether length and $f(s)=1 / \alpha^{2}\left(x^{\prime}-1\right) / x^{\prime}$ is the adimensional variable tether tension. Furthermore, the variables have been adimensionalized with respect to $\ell$ and $n$ in space and time, respectively, and ()$^{\prime}$ denotes derivation with respect to $s$ and ()$^{\bullet}$ with respect to $t$.

It has to be pointed out that even if the control force is linear in the displacement variables, its effect in the transversal motion is nonlinear according to the fact that it results in an additional longitudinal component. In particular, it produces new quadratic terms which are similar to those of gyroscopic forces.

It is useful to introduce a more compact expression of the equations of motion and boundary conditions which can be written as:

$$
\begin{array}{r}
{\left[M(\ddot{v})+L_{v}(v)+B(v, \dot{v})+C(w, \dot{w})+R\left(v, \dot{v}_{e}\right)\right] \times \delta v=0} \\
{\left[M(\ddot{w})+L_{w}(w)+D(w, \dot{v})+R\left(w, \dot{v}_{e}\right)\right] \times \delta w=0}
\end{array}
$$

where $M$ and $L$ are linear integro-differential operators of $s$ and $B, C, D$, $R$ are bilinear integro-differential operators, whose explicit expressions can be obtained from comparisons of (1) and (2) with (4) after some manipulations [15].

The large amplitude oscillations are coupled and all the displacement components are involved in the motion. The nonlinear terms are such that the motion occurs in the orbit plane if zero initial conditions on the $w$ displacements are given; the dual situation is not true, as the out-of-plane motion will always force the transversal in-plane oscillations. 


\section{STATIONARY OSCILLATIONS}

It has been observed in [16] that both in-plane and out-of-plane nonlinear vibrations with a prevailing component can be satisfactorily studied by means of a truncated two-modes model. The control force does not change this peculiarity. Indeed, when an oscillation with a prevailing inplane mode with frequency $\omega_{k}$ is considered, the nonlinear terms $B(v, \dot{v})+$ $R\left(v, \dot{v}_{e}\right)$ in (4a) forces other modes among which the only important one to be retained is that with frequency $\omega_{j}$ close to $2 \omega_{k}$. On the other side, if an out-of-plane mode with frequency $\omega_{k}$ is the prevailing component, the nonlinear term $C(w, \dot{w})$ in (4a) forces the arising of in-plane modes, of which, similarly to the in-plane oscillation, the most important one is that with frequency $\omega_{j}$ close to $2 \omega_{k}$.

When the stationary solutions are sought, the harmonic balance method can be conveniently used since it remains valid in conditions of internal resonance.

\subsection{In-plane oscillations}

As pointed out, a periodic solution with only two components with nonlinear frequencies $\Omega_{k}$ and $2 \Omega_{k}$ is suitable to study the steady-state inplane oscillations:

$$
\begin{aligned}
v(s, t)= & \varphi_{k}(s)\left[A_{k} \cos \Omega_{k} t+B_{k} \sin \Omega_{k} t\right] \\
& +\varphi_{j}(s)\left[A_{j} \cos 2 \Omega_{k} t+B_{j} \sin 2 \Omega_{k} t\right]
\end{aligned}
$$

where $\varphi_{k}$ and $\varphi_{j}$ are the linear modes with frequency $\omega_{k}$, close to $\Omega_{k}$ for small amplitudes, and $\omega_{j}$ which differs from $2 \omega_{k}$ for a small quantity $\sigma_{k}$.

By substituting the approximate expression (5) of $v(s, t)$ in (4), performing all integrations and equating terms with the same harmonics, the following equations are obtained:

$$
\begin{array}{r}
\left(\omega_{k}^{2}-\Omega_{k}^{2}\right) m_{k} A_{k}+\Omega_{k}\left(b_{k k j}+r_{k k j}-\frac{1}{2} r_{k j k}\right)\left(A_{k} B_{j}-A_{j} B_{k}\right)=0 \\
\left(\omega_{k}^{2}-\Omega_{k}^{2}\right) m_{k} B_{k}-\Omega_{k}\left(b_{k k j}+r_{k k j}-\frac{1}{2} r_{k j k}\right)\left(A_{k} A_{j}+B_{k} B_{j}\right)=0 \\
\left(\omega_{j}^{2}-4 \Omega_{k}^{2}\right) m_{j} A_{j}+\frac{1}{2} \Omega_{k}\left(b_{j k k}+r_{j k k}\right) A_{k} B_{k}=0 \\
\left(\omega_{j}^{2}-4 \Omega_{k}^{2}\right) m_{j} B_{j}+\frac{1}{2} \Omega_{k}\left(b_{j k k}+r_{j k k}\right)\left(B_{k}^{2}-A_{k}^{2}\right)=0
\end{array}
$$

where coefficients of these equations and those following are given in Appendix B.

Having assumed a velocity dependent linear control force, these equations have the same structure of those without control and only the coef- 
ficients are affected by this force. The solution can be expressed, in any case, by only two amplitudes; the case with $A_{j}=B_{k}=0$ is presented. From (6a) the frequency-amplitude relationship is determined:

$$
\Omega_{k} \simeq \omega_{k}\left(1+\frac{b_{k k j}+r_{k k j}-1 / 2 r_{k j k}}{\omega_{k} m_{k}} B_{j}\right)^{1 / 2},
$$

while (6d), together with (7) and $\sigma_{k}=2 \omega_{k}-\omega_{j}$, gives the $A_{k}-B_{j}$ relationship, which describes the modification of the shape with oscillation amplitudes:

$$
\frac{b_{k k j}+r_{k k j}-1 / 2 r_{k j k}}{m_{k}} B_{j}^{2}+\sigma_{k} B_{j}+\frac{b_{k k j}+r_{k k j}}{8 m_{j}} A_{k}^{2}=0 .
$$

\subsection{Out-of-plane oscillations}

The monofrequent out-of-plane oscillation of nonlinear frequency $\Omega_{k}$ near to the linear frequency $\omega_{k}$, as in the uncontrolled problem, is characterized by a prevailing out-of-plane component and a forced in-plane one with frequency $2 \Omega_{k}$ close to $\omega_{j}$ :

$$
\begin{aligned}
& v(s, t)=\varphi_{j}(s)\left[A_{j} \cos 2 \Omega_{k} t+B_{j} \sin 2 \Omega_{k} t\right] \\
& w(s, t)=\psi_{k}(s)\left[A_{k} \cos \Omega_{k} t+B_{k} \sin \Omega_{k} t\right],
\end{aligned}
$$

where $\psi_{k}$ is the out-of-plane linear mode with frequency $\omega_{k}$.

Following the harmonic balance procedure, a system of four equations similar to (6) is obtained whose solution gives the frequency-amplitude relation

$$
\Omega_{k} \simeq \omega_{k}\left(1+\frac{d_{k k j}+r_{k k j}-1 / 2 r_{k j k}}{\omega_{k} m_{k}} B_{j}\right)^{1 / 2}
$$

and the amplitude relationship $B_{j}-A_{k}$

$$
\frac{d_{k k j}+r_{k k j}-1 / 2 r_{k j k}}{m_{k}} B_{j}^{2}+\sigma_{k} B_{j}+\frac{c_{k k j}}{8 m_{j}} A_{k}^{2}=0 .
$$

Even for out-of-plane oscillations, the control force does not change the kind of solution with respect to the uncontrolled case [15], but it only modifies the coefficients. 


\section{STABILITY OF STATIONARY OSCILLATIONS}

A stability analysis of the stationary solutions is performed by introducing in (4) a perturbed steady-state solution

$$
v(s, t)=v_{s}(s, t)+\eta(s, t) \quad w(s, t)=w_{s}(s, t)+\zeta(s, t) .
$$

Linearizing the motion equations with respect to $\eta$ and $\zeta$ and describing each component of the disturbance by one suitably selected mode

$$
\eta(s, t)=\phi_{i}(s) \eta(t) \quad \zeta(s, t)=\psi_{l}(s) \zeta(t)
$$

the use of the Galerkin technique leads to the variational equations

$$
\begin{aligned}
& {\left[\begin{array}{cc}
m_{1} & 0 \\
0 & m_{\dot{2}}
\end{array}\right]\left\{\begin{array}{l}
\ddot{\eta} \\
\ddot{\zeta}
\end{array}\right\}+\left(\left[\begin{array}{cc}
0 & g_{12} \\
-g_{12} & 0
\end{array}\right]+\left[\begin{array}{cc}
e_{11} & 0 \\
e_{21} & 0
\end{array}\right]\right)\left\{\begin{array}{l}
\dot{\eta} \\
\dot{\zeta}
\end{array}\right\}} \\
& +\left(\left[\begin{array}{cc}
k_{1} & 0 \\
0 & k_{2}
\end{array}\right]+\left[\begin{array}{cc}
f_{11} & f_{12} \\
0 & f_{22}
\end{array}\right]+\left[\begin{array}{cc}
c_{11} & 0 \\
0 & c_{22}
\end{array}\right]\right)\left\{\begin{array}{l}
\eta \\
\zeta
\end{array}\right\}=\left\{\begin{array}{l}
0 \\
0
\end{array}\right\}
\end{aligned}
$$

For in-plane oscillations, (14) are uncoupled and, consequently, in-plane and out-of-plane disturbances can be separately applied, while coupled perturbations must be introduced for analysing stability of out-of-plane oscillations.

In the following the stability of planar oscillations for out-of-plane disturbances is investigated. In this case, the stability is governed by the only (14b), which, after explicit calculation of the coefficients and introducing a new time variable $\tau=\left(\Omega_{k} / 2\right) t+\pi / 4$, becomes

$$
\ddot{\zeta}(\tau)+\left(\delta^{2}+\epsilon_{1} \cos 2 \tau+\epsilon_{2} \cos 4 \tau\right) \zeta(\tau)=0,
$$

where

$$
\delta^{2}=\frac{2 \omega_{l}}{\Omega_{k}} \quad \epsilon_{1}=4 \frac{A_{k}}{m_{l} \Omega_{k}}\left(d_{l l k}+r_{l l k}\right) \quad \epsilon_{2}=-8 \frac{B_{j}}{m_{l} \Omega_{k}}\left(d_{l l j}+r_{l l j}\right) .
$$

Equation (15) has periodic coefficients of frequency 2 and, according to the Floquet theory, boundaries of the first instability region are associated with periodic solutions of frequency 1. Expanding $\zeta(\tau)$ in series of $\sin \tau$, $\cos \tau, \sin 3 \tau, \cos 3 \tau$, substituting in (15) and vanishing the terms with same harmonics, two uncoupled eigenvalues problems are obtained, which give 
TABLE 1

LINEAR FREQUENCIES OF TRANSVERSAL MODES (PENDULUM

TYPE $k=0$, FLEXIBLE MODES $k>0$ )

\begin{tabular}{lrrrrr}
\hline & $k=0$ & $k=1$ & $k=2$ & $k=3$ & $k=4$ \\
\hline$\omega_{k}^{v}$ & 1.736 & 23.022 & 45.845 & 68.712 & 91.590 \\
$\omega_{k}^{w}$ & 2.004 & 23.044 & 45.856 & 68.719 & 91.595 \\
$p_{k}$ & 0.238 & 3.160 & 6.292 & 9.341 & 12.571 \\
\hline
\end{tabular}

the boundaries of the unstable region

$$
\delta^{2}=1 \pm \frac{\epsilon_{1}}{2}-\frac{1}{32^{2}}\left(\epsilon_{1} \pm \epsilon_{2}\right)
$$

The primary instability region emanates from $\Omega_{k}=2 \omega_{l}$. For a sample system, Table 1 shows that it can easily occur for each in-plane mode, as there is always a lower out-of-plane mode with almost half frequency, apart from the first one.

It is worthwhile to consider the region of secondary instability, for which $\zeta(\tau)$ is expanded in series of $\sin 2 \tau, \cos 2 \tau, \sin 4 \tau, \cos 4 \tau$, plus a constant term. The boundaries of the unstable region are

$$
\delta^{2}=4+\frac{\epsilon_{2}}{2}+\frac{5}{48} \epsilon_{1}^{2} \quad \delta^{2}=4+\frac{\epsilon_{2}}{2}-\frac{1}{48} \epsilon_{1}^{2}
$$

and emanate from $\Omega_{k}=\omega_{l}$. This condition is verified, for example, for the basic case of the first in-plane mode perturbed by the first out-ofplane mode.

Numerical results have been obtained for a sample string-satellite system whose parameters are given in Appendix B. Figure 2 shows the primary instability regions of steady in-plane oscillations and a prevailing second in-plane mode $(k=2, j=4)$ perturbed by the resonant first out-of-plane mode $(l=1)$, either with or without control. It can be observed that the control force narrows the instability region and, even if the frequencyamplitude curve is more bent, the critical amplitude is higher; little modification results in the amplitude relationship $A_{k}-B_{j}$ (Figure 2a). As the value of the control gain $k_{c}$ raises, the region becomes narrower and the critical amplitude greater, while the corresponding nonlinear frequency $\Omega_{k}$ becomes closer to the linear one. For a prevailing first in-plane mode $(k=1, j=2)$ perturbed by the first out-of-plane resonant mode $(l=1)$, Figure 3 shows the secondary instability region; the control force narrows the unstable region but, on the other side, the curve is more bent. In this 


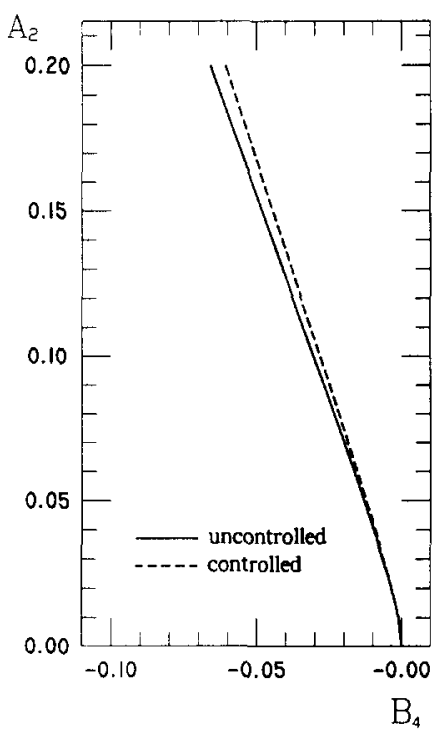

(a)

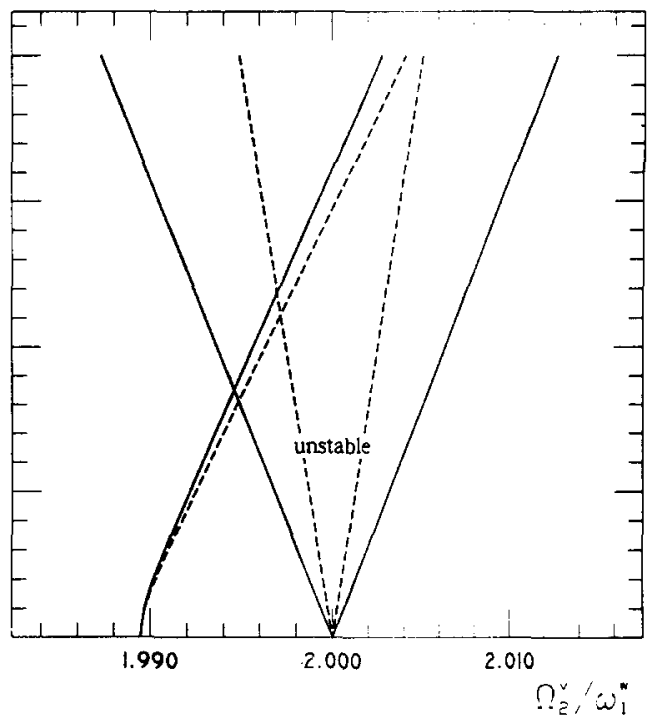

(b)

FIG. 2. Primary instability of the second in-plane mode: (a) amplitudes; (b) frequency-amplitude curve and instability region.

case, the two opposite effects result in a smaller improvement of the critical condition but, nevertheless, are effective, since the length of the unstable branch of the curve is reduced.

\section{IN-PLANE PERIODICALLY MODULATED AMPLITUDE OSCILLATIONS}

A three-mode model is used to study the in-plane motion, without limiting the analysis to the case of constant amplitude, and the evolution of the spatial motion when the unstable in-plane oscillations are perturbed outof-plane. To this scope the amplitude modulations equations are obtained from the second-order perturbation equations.

By introducing new time scale $t_{n}=\epsilon^{n} t(n=0,1, \ldots)[17]$ and expanding displacements $v(s, t)$ and $w(s, t)$ in two-term series of the perturbation parameter $\epsilon$, the two linear system at order $\epsilon$ and $\epsilon^{2}$ are obtained, respectively,

$$
\begin{array}{r}
{\left[M\left(d_{00}^{2} v_{0}\right)+L_{v}\left(v_{0}\right)\right] \times \delta v=0} \\
{\left[M\left(d_{00}^{2} w_{0}\right)+L_{w}\left(w_{0}\right)\right] \times \delta w=0}
\end{array}
$$




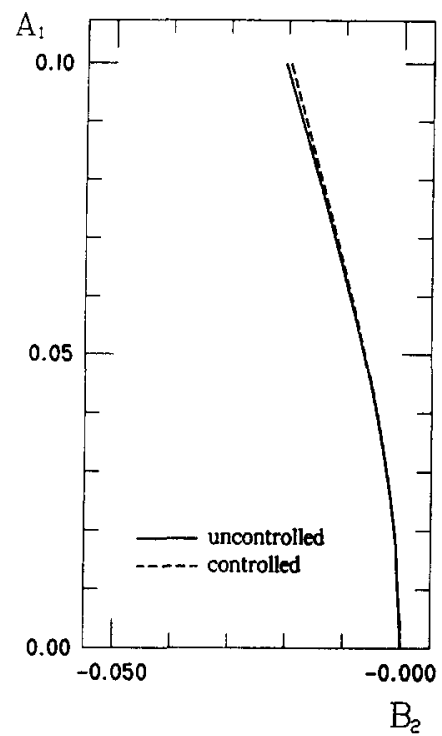

(a)

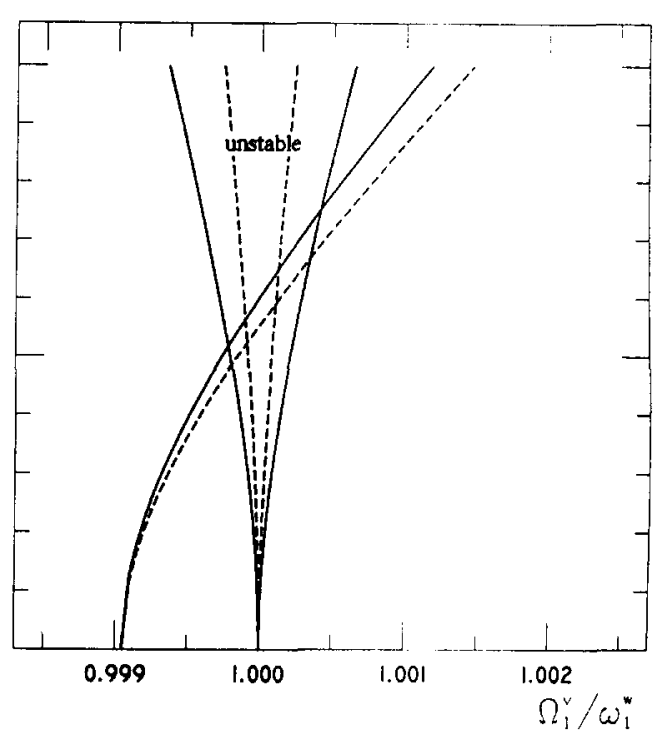

(b)

FIG. 3. Secondary instability of the first in-plane mode: (a) amplitudes; (b) frequency-amplitude curve and instability region.

$$
\begin{aligned}
& {\left[M\left(d_{00}^{2} v_{1}\right)+L_{v}\left(v_{1}\right)\right] \times \delta v=-\left[2 M\left(d_{01}^{2} v_{0}\right)+B\left(v_{0}, d_{0} v_{0}\right)\right.} \\
& \left.+C\left(w_{0}, d_{0} w_{0}\right)+R\left(v_{0}, d_{0} v_{e 0}\right)\right] \times \delta v \\
& {\left[M\left(d_{00}^{2} w_{1}\right)+L_{w}\left(w_{1}\right)\right] \times \delta w=-\left[2 M\left(d_{01}^{2} w_{0}\right)+D\left(w_{0}, d_{0} v_{0}\right)\right.} \\
& \left.+R\left(w_{0}, d_{0} v_{e 0}\right)\right] \times \delta w,
\end{aligned}
$$

where $d_{n}=\partial / \partial t_{n}$ and $d_{m n}=\partial^{2} / \partial t_{m} \partial t_{n}$.

In this problem the two in-plane components $k$ th and $j$ th are in primary resonance conditions $\left(2 \omega_{k}=\omega_{j}+\epsilon \sigma_{k}\right)$, while the out-of-plane mode lth can be taken in primary $\left(2 \omega_{l}=\omega_{k}+\epsilon \sigma_{l}\right)$ or in secondary $\left(2 \omega_{l}=\omega_{j}+\epsilon \sigma_{l}\right)$ resonance conditions. In both cases the generating solution is described by three components:

$$
\begin{aligned}
& v_{0}(s, t)=A_{k}\left(t_{1}\right) \varphi_{k}(s) e^{i \omega_{k} t_{0}}+A_{j}\left(t_{1}\right) \varphi_{j}(s) e^{i \omega_{j} t_{0}}+\text { c.c. } \\
& w_{0}(s, t)=A_{l}\left(t_{1}\right) \psi_{l}(s) e^{i \omega_{l} t_{0}}+\text { c.c. }
\end{aligned}
$$

where c.c. stands for complex conjugate. Equations (21) are used in (20) where $\delta v=\varphi_{k}, \delta v=\varphi_{j}$, and $\delta w=\psi_{l}$ are taken successively. By zeroing secular terms, making use of the polar form to represent the complex 
amplitudes, $A_{h}=1 / 2 a_{h}\left(t_{1}\right) e^{i \vartheta_{h}\left(t_{1}\right)}$, and separating real and imaginary parts, six equations in the three amplitudes $a_{h}$ and the three phases $\vartheta_{h}$ are obtained.

The introduction of suitable new variables $\gamma_{k}$ and $\gamma_{l}$ makes it possible to reduce previous system to an autonomous system of five equations. For an out-of-plane mode in primary resonance condition they read:

$$
\begin{aligned}
a_{k}^{\prime} & =-\left(b_{k}+r_{k}\right) a_{k} a_{j} \cos \gamma_{k}+c a_{l}^{2} \cos \gamma_{l} \\
a_{j}^{\prime} & =-\left(b_{j}+r_{j}\right) a_{k}^{2} \cos \gamma_{k} \\
a_{l}^{\prime} & =-(d+s) a_{k} a_{l} \cos \gamma_{l} \\
a_{k} a_{j} \gamma_{k}^{\prime} & =\left[b_{j} a_{k}^{3}-2\left(b_{k}+r_{k}\right) a_{k} a_{j}^{2}\right] \sin \gamma_{k}+c a_{j} a_{l}^{2} \sin \gamma_{l}+\sigma_{k} a_{k} a_{j} \\
a_{l} a_{k} \gamma_{l}^{\prime} & =\left[2(d+s) a_{l} a_{k}^{2}-c a_{l}^{3}\right] \sin \gamma_{l}-\left(b_{k}+r_{k}\right) a_{l} a_{k} a_{j} \sin \gamma_{k}+\sigma_{l} a_{l} a_{k},
\end{aligned}
$$

where

$$
\gamma_{k}=2 \vartheta_{k}-\vartheta_{j}+\sigma_{k} t_{1} \quad \gamma_{l}=2 \vartheta_{l}-\vartheta_{k}+\sigma_{l} t_{1} .
$$

Similar equations are obtained for secondary resonant out-of-plane mode. The fixed points of (22) include all the steady-state oscillations obtained by harmonic balance method in the previous section. However, the threemode model is not able to describe accurately the evolution of disturbed, steady or periodic solutions with a prevailing out-of-plane mode $l$ th and a companion $k$ th. Indeed, as pointed out before, the disturbance must be described at least by two modes. Thus, in the following, the attention is focused on the planar motion described by the three equations (22a), (22b), (22d) with $a_{l}=0$.

In both resonant cases, a first integral of motion of the kind $a_{k}^{2}+$ $\left(b_{k} / b_{j}\right) a_{j}^{2}=E=$ const can be easily deduced from the first two equations. This implies that the trajectories of motion run on a cylinder in the $a_{k}, a_{j}, \gamma_{k}$ space; in Figure 4, for two different in-plane oscillations, the curved surface is extended onto a plane where the angle $\beta=\arctan \left(a_{k} / a_{j}\right)$ is introduced and the cyclic variable $\gamma_{k}$ is represented in $[-\pi, \pi]$. Three different stationary solutions are found: a one-component $S_{0}\left(a_{k}=0, a_{j} \neq 0\right.$, i.e. $\beta=0$ ) and the two two-components $S_{1}$ and $S_{2}$, the former with the prevailing $a_{k}$ and the latter $a_{j}$. However, the attention is restricted to the motions around $S_{1}$ with most of initial energy in $a_{k}$. The amplitude periodic solutions are described by closed and open trajectories; notable amplitude and phase modulations occur on most of them, except the closed trajectories just in a small region around $S_{1}$.

Since (22) cannot be put in normal form when $a_{l}=0$, they cannot be used for the stability analysis. A suitable transformation of variables has 

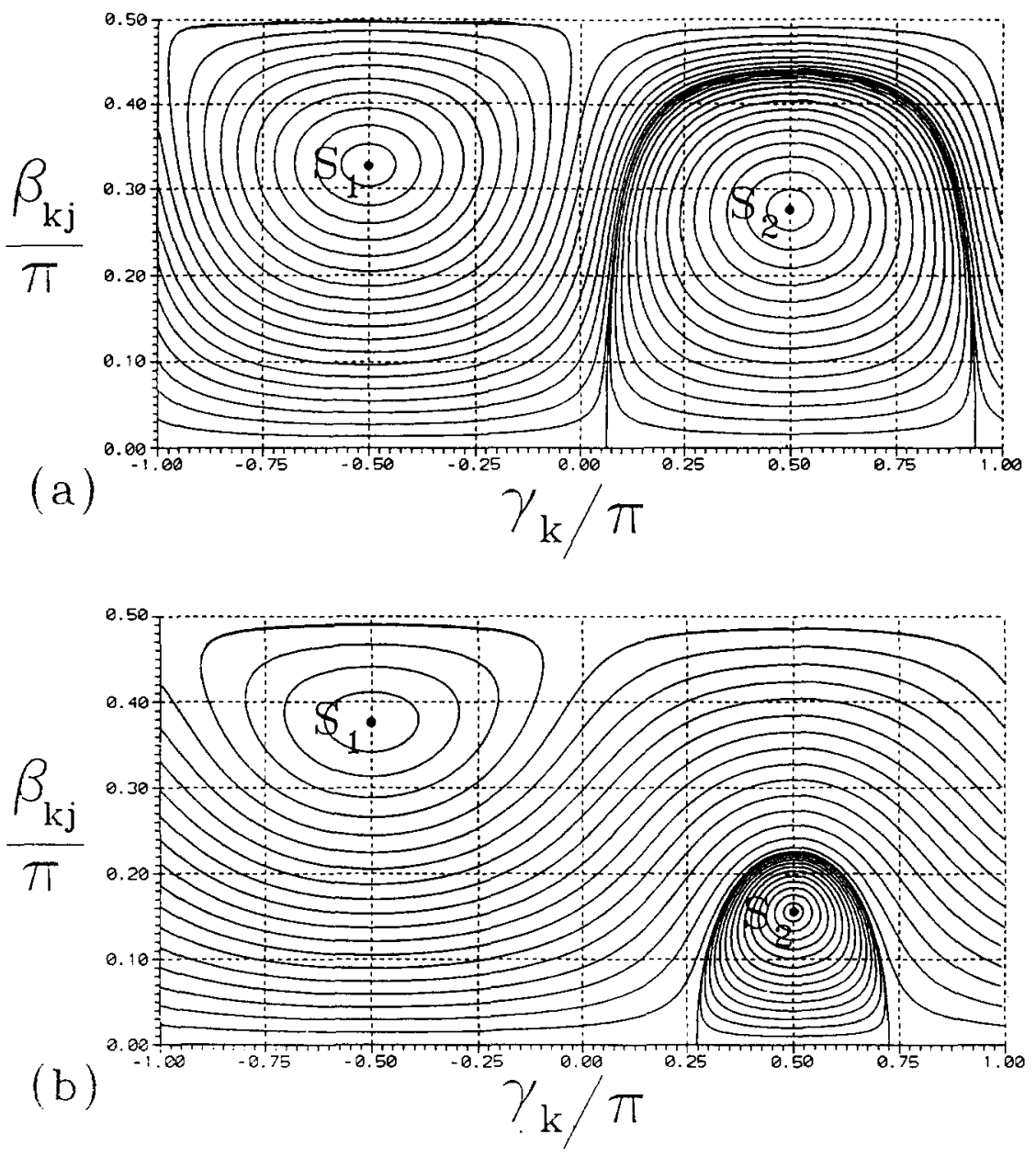

FIG. 4. Trajectories of nonstationary two-modes motion: (a) $k=2, j=4$; (b) $k=1, j=2$. 

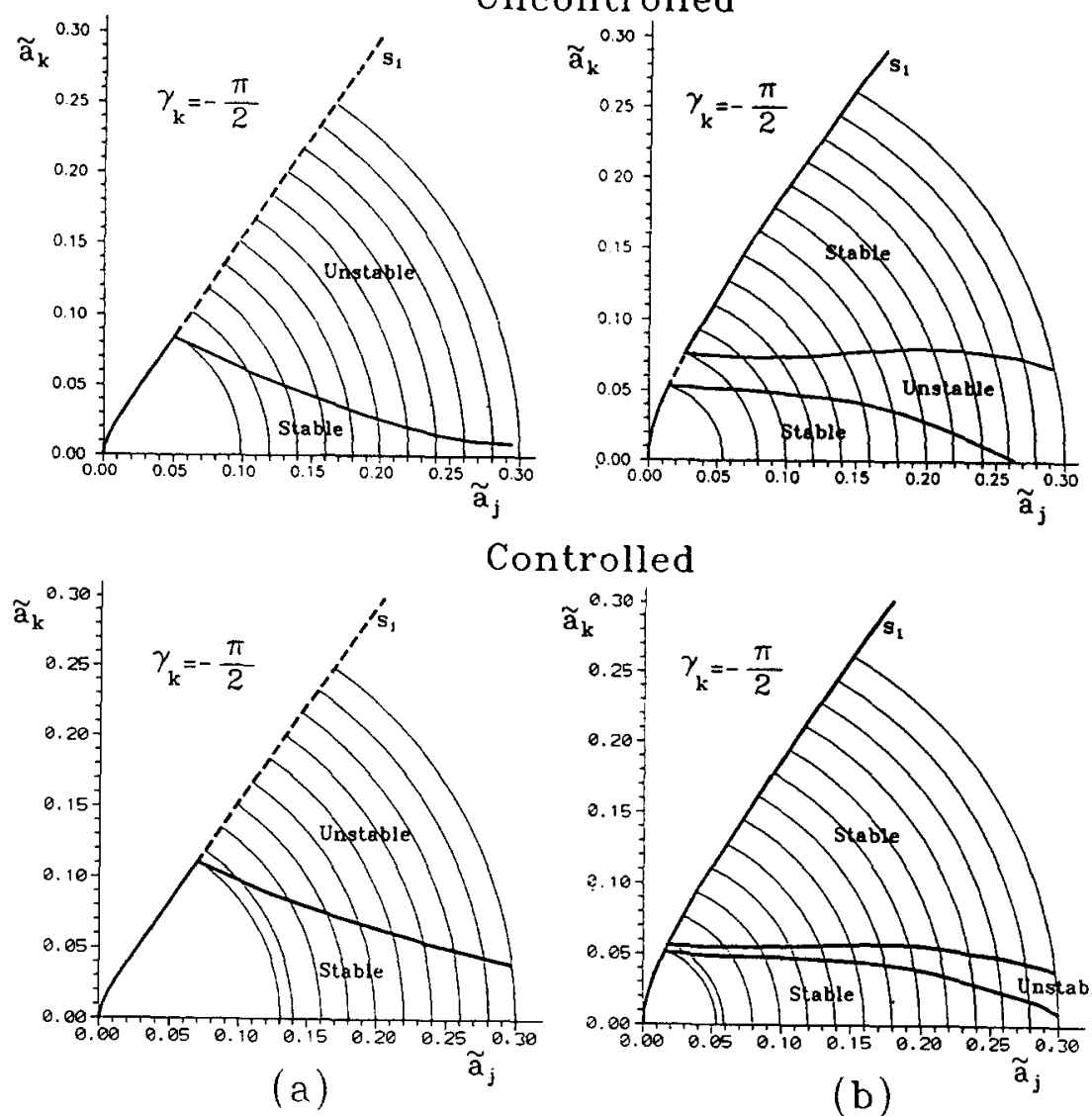

Controlled

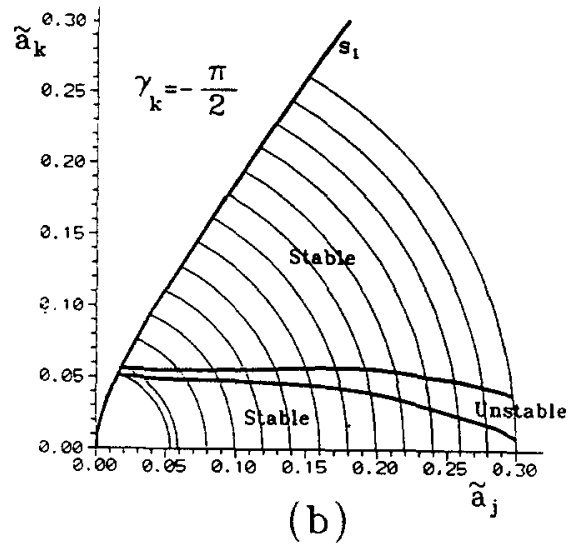

FIG. 5. Instability regions of the trajectories on the section of the normalized amplitudes $\left(\tilde{a}_{h}=a_{h} / E\right)$ at $\gamma_{k}=\pi / 2$ : (a) primary and (b) secondary resonance.

been proposed in [16] which makes it possible to reconduct the stability investigation to the solution of classical variational equations with periodic coefficients. The results are summarized in Figure 5 which represents a section of the cylinder at $\gamma_{k}=-\pi / 2$ crossing $S_{1}$ for a primary (a) and secondary (b) resonant conditions both for uncontrolled and controlled cases. The stationary solutions, whose behavior is the same as discussed in Section 3, are the first to become unstable. For increasing levels of energy the instability propagates toward external trajectories and the unstable region becomes wider in the primary resonance. In the secondary resonance the picture is more complex since the stationary solutions lose and regain sta- 


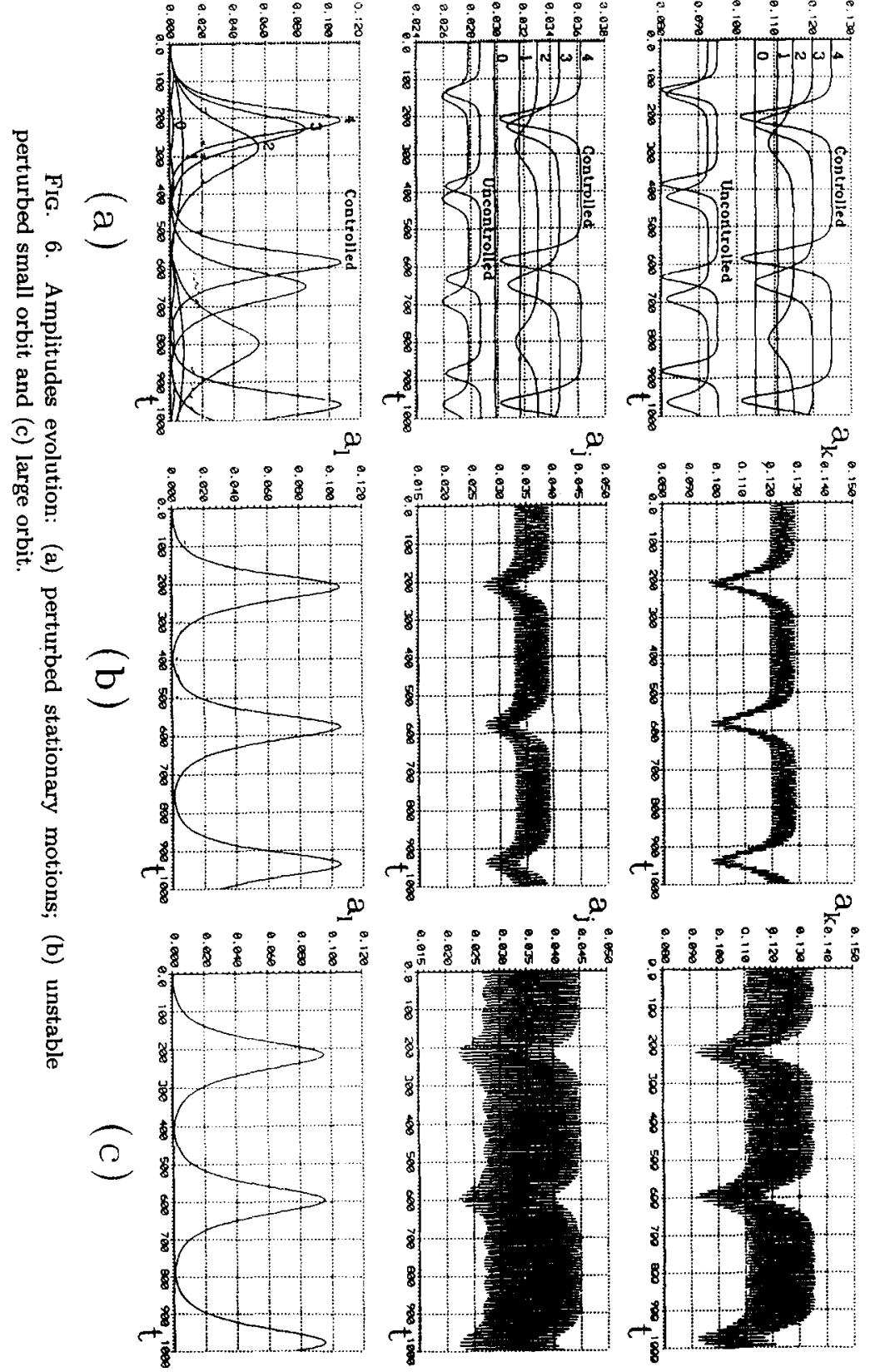


bility. Thus, at a certain level of energy a small region of periodic orbits are unstable and all the others are stable, while at higher level orbits close to the steady solution are stable, then a region of unstable orbits is found and further trajectories are stable again. The effect of the control force on the stability of stationary oscillations has been already discussed in Section 4; its effect on the stability of periodic trajectories is evident in Figure 5 from comparison with the uncontrolled case.

The evolution of unstable in-plane orbits perturbed out-of-plane is obtained by numerically solving the system (22). The qualitative behavior of the controlled and uncontrolled cases is practically the same, maintaining the general consideration that the stable region of controlled system is by far wider. The response of unstable stationary solutions for increasing initial amplitude is illustrated in Figure 6a; the motion becomes periodic with an energy transfer from planar modes to the out-of-plane mode, which is faster as the energy of the steady oscillation is higher. In Figure $6 \mathrm{~b}$ and $6 \mathrm{c}$ the evolution of two orbits around $S_{1}$ (Figure 4a) with the same energy of the steady solution denoted by number 4 (Figure 6a) are reported. For small orbits close to steady solutions (b) the mean values of the amplitude have a trend very similar to that of steady solution, while for larger orbits (c) which are close to the stable region the mean values tend to be constant. Substantially, in the first case a little fast modulation of amplitude is associated to large slow energy transfer, while in the other case large fast modulation occurs with lower energy transfer among in-plane and out-of-plane modes.

\section{CONCLUSIONS}

The nonlinear dynamics of a tethered satellite system in the deployed configuration offers interesting points to be investigated, notwithstanding it is characterized by weak nonlinearities. This is due to the fact that the system exhibits quadratic nonlinearities and the frequencies of flexible modes are almost in integer ratio. Accordingly, the system practically works in conditions of internal resonance which produce instability of the motion.

The stabilizing effect of a longitudinal control force has been investigated in this paper. The structure of nonlinear terms is such that the transversal displacement component in the orbit plane is always present, either as a prevailing component in the planar motion or as a companion component when the prevailing one is an out-of-plane mode. This makes it possible to use a control force which is a function of in-plane component only.

It is shown that the assumed control force, with a realistic value of the 
gain constant, is effective in reducing the instability region of stationary and periodically modulated amplitude oscillations perturbed by primary and secondary internally resonant components.

\section{APPENDIX A}

The coefficients introduced in (6) and (10), (11) are defined as follows:

$$
\begin{array}{lll}
m_{k}=M\left(h_{k}\right) \times h_{k} & r_{i j k}=R\left(h_{j}, \varphi_{k}\right) \times h_{i} & \text { with } h=\varphi, \psi \\
b_{i j k}=B\left(\varphi_{j}, \varphi_{k}\right) \times \varphi_{i} & c_{i j k}=C\left(\psi_{j}, \psi_{k}\right) \times \varphi_{i} & d_{i j k}=D\left(\psi_{j}, \varphi_{k}\right) \times \psi_{i} .
\end{array}
$$

In (14) are introduced the following positions:

$$
\begin{array}{ll}
m_{1}=M\left(\varphi_{i}\right) \times \varphi_{i} & m_{2}=M\left(\psi_{l}\right) \times \psi_{l} \\
k_{1}=L_{v}\left(\varphi_{i}\right) \times \varphi_{i} & k_{2}=L_{w}\left(\psi_{l}\right) \times \psi_{l} \\
g_{11}=B\left(v_{s}, \varphi_{i}\right) \times \varphi_{i} & g_{12}=C\left(w_{s}, \psi_{l}\right) \times \varphi_{i} \\
& g_{21}=D\left(w_{s}, \varphi_{i}\right) \times \psi_{l}=-g_{12} \\
f_{11}=B\left(\varphi_{i}, \dot{v}_{s}\right) \times \varphi_{i} & f_{12}=C\left(\psi_{l}, \dot{w}_{s}\right) \times \varphi_{i} \\
& f_{22}=D\left(\dot{\psi}_{l}, \dot{v}_{s}\right) \times \psi_{l} \\
e_{11}=R\left(v_{s}, \varphi_{i e}\right) \times \varphi_{i} & e_{21}=R\left(w_{s}, \varphi_{i e}\right) \times \varphi_{i} \\
c_{11}=R\left(\varphi_{i}, \dot{v}_{s e}\right) \times \varphi_{i} & c_{22}=R\left(\psi_{j}, \dot{v}_{s e}\right) \times \psi_{j} .
\end{array}
$$

The coefficients in (22) are

$$
\begin{aligned}
& b_{k}=\frac{1}{4 m_{k}}\left(b_{k j k}-\frac{\omega_{j}}{\omega_{k}} b_{k k j}\right) b_{j}=\frac{1}{4 m_{j}} \frac{\omega_{k}}{\omega_{j}} b_{j k k} \\
& r_{k}=\frac{1}{4 m_{k}}\left(r_{k j k}-\frac{\omega_{j}}{\omega_{k}} r_{k k j}\right) \quad r_{j}=\frac{1}{4 m_{j}} \frac{\omega_{k}}{\omega_{j}} r_{j k k} \\
& c=-\frac{1}{4 m_{k}} \frac{\omega_{l}}{\omega_{k}} c_{k l l} \quad d=\frac{1}{4 m_{l}} \frac{\omega_{k}}{\omega_{l}} d_{l l k} \quad s=\frac{1}{4 m_{l}} \frac{\omega_{k}}{\omega_{l}} r_{l l k} .
\end{aligned}
$$

\section{APPENDIX B}

The following values of the parameters are assumed for the sample system:

$$
\begin{array}{lll}
n^{2}=1.35 \times 10^{-6} \mathrm{~s}^{-2} & a=6657 \mathrm{~km} & \ell=5 \mathrm{~km} \\
M=10^{5} \mathrm{~kg} & m=500 \mathrm{~kg} & \mu=5.76 \times 10^{-6} \mathrm{kgm}^{-2} \\
E=7 \times 10^{10} \mathrm{Nm}^{-2} & A=4 \times 10^{-6} \mathrm{~m}^{2} & K_{c}=29.894 \mathrm{Nm}^{-1} \mathrm{~s} .
\end{array}
$$


The corresponding nondimensional parameters (3) are: $\alpha^{2}=6.94 \times 10^{-7}$, $\gamma=0.0576, \xi=7.51 \times 10^{-4}$, and $k_{c}=10$.

The first five in-plane and out-of-plane time frequencies $\omega_{k}^{v}$ and $\omega_{k}^{w}$ are given in Table 1 together with the spatial frequencies $p_{k}$ of the associated modal shapes, $\varphi_{k}(s)=\psi_{k}(s) \simeq \sin p_{k} s[5]$.

This research is partially supported by Italian Ministry of University and Scientific Technological Research, 1992.

\section{REFERENCES}

1 A. K. Misra and V. J. Modi, A general dynamical model for the space shuttle based tethered subsatellite system, in Astrodynamics 1979-Advances in the Astronautical Sciences, Vol. 40, 1979, pp. 537-557.

2 A. K. Banerjee and T. R. Kane, Tether development dynamics, J. Astronaut. Sci. 30:347-365 (1982).

3 V. V. Beletskii and E. M. Levin, Dynamics of the orbital cable system, Acta Astronaut. 12:285-291 (1985).

4 A. H. von Flotow, Some approximations for the dynamics of tethered spacecraft, J. Guidance, Control, Dynamics 11:357-364 (1988).

5 M. Pasca, M. Pignataro, and A. Luongo, Three-dimensional vibrations of tethered satellite systems, J. Guidance, Control, Dynamics 14:312-320 (1991).

6 V. J. Modi, G. Chang-Fu, A. K. Misra, and D. M. Xu, On the control of the space shuttle based tethered systems, Acta Astronaut. 9:437-443 (1982).

7 Liangdong Liu and P. M. Bainum, Effect of tether flexibility on the tethered shuttle subsatellite stability and control, J. Guidance, Control, Dynamics $12: 866,873$ (1989).

8 D. J. Pines, A. H. von Flotow, and D. C. Redding, Two nonlinear control approaches for retrieval of a thrusting tethered subsatellite, J. Guidance, Control, Dynamics 13:651,658 (1990).

9 M. Pasca, A. Luongo, M. Pignataro, and F. Vestroni, Free Dynamics of the Shuttle-Tethered-Satellite System, Department of Structural and Geotechnical Engineering, University of Rome "La Sapienza," Rome, Italy, No. 3, 1987.

10 F. Vestroni and A. Luongo, Perturbation analysis of finite oscillations of an orbiting string, in Trends in Applications of Mathematics to Mechanics (W. Schneider, H. Troger, F. Zeigler, Eds.), Longman Scientific and Technical, 1991.

11 A. H. Nayfeh and B. Balachadran, Modal interactions in dynamical and structural systems, Appl. Mech. Rev. 42:175-202 (1989).

12 A. K. Bajaj and J. M. Johnson, On the amplitude dynamics and crisis in resonant motion of stretched strings, Phil. Trans. Roy. Soc. London, 338:1-41 (1992). 
13 C. L. Lee and N. C. Perkins, Three-dimensional oscillations of suspended cables involving simultaneous internal resonances, in ASME Winter Annual Meeting, 1992.

14 R. A. Raouf and A. H. Nayfeh, Autoparametric resonances in infinitely long cylindrical shells, Computers and Structures 35:163-173 (1990).

15 A. Luongo and F. Vestroni, Nonlinear free periodic oscillations of a tethered satellite system, J. Sound and Vibrations, 175(3):299-315 (1994).

16 A. Luongo and F. Vestroni, Bifurcation and Stability of Nonstationary Planar Motions of an Orbiting String, Report DISAT, University of L'Aquila, L'Aquila, Italy, 1993.

17 A. H. Nayfeh, Perturbation Methods, John Wiley and Sons, New York, 1973. 